\title{
Die AlLEINHERRSCHAFT DES DeMOTELES Auf SAMOS
}

\author{
F. X. Ryan
}

\begin{abstract}
There is now an overwhelming consensus in favor of the view that the monarchy attested for Demoteles on Samos was a tyranny. The key piece of evidence, however, is ambiguous. When interpreted one way, another solution is equally probable, and when interpreted another way, this other solution alone is possible.
\end{abstract}

Der samische Alleinherrscher Demoteles wird in den uns erhaltenen Quellen nur ein einziges Mal erwähnt, ${ }^{1}$ und auch dann nur deswegen, weil sein Tod als Orientierungshilfe dienen sollte: nach seiner Ermordung, als die Grundadligen das Land regierten, hätten die Megarer die Bewohner der samischen Pflanzstadt Perinthos überfallen; die Grundadligen schickten daraufhin eine Flottenmannschaft nach Perinthos, welche die Megarer besiegte (Plut. QG $57=$ Mor. 303E-304C). Da aber die Überlieferung sonst kein Wort über Demoteles verliert, vermag die Nachricht nicht mehr das zu tun, was sie tun sollte, nämlich den Krieg gegen Megara datieren, wir müssen vielmehr über diesen Krieg die Alleinherrschaft des Demoteles datieren, von der allein dieser Datierungsversuch uns in Kenntnis setzt. Das eigentliche Ziel dieser Schrift, auf die Art der Alleinherrschaft des Demoteles näher einzugehen, werden wir erst verfolgen, nachdem wir der leidigen Frage nach der Datierung derselben nachgegangen sind.

\section{Die Zeit der Alleinherrschaft des Demoteles}

Die Tatsache, daß der Tod des Demoteles noch vor dem Krieg gegen die Megarer liegt, ${ }^{2}$ ist zunächst wenig hilfreich, denn auch der Angriff der Megarer auf Perinthos wird anderweitig nicht erwähnt. Der Krieg gehört auf jeden Fall in die Zeit vor der Eroberung Ioniens durch die Perser im Jahre 546 v. Chr., ${ }^{3}$ und wird gelegentlich ziemlich spät angesetzt, in die Jahre um 570-560. ${ }^{4}$ Datiert wird der Krieg aber auch um 600 oder in die 590er Jahre $^{5}$ in der Annahme, daß Megara wegen seiner Kolonie Selymbria sogleich versucht

\footnotetext{
${ }^{1}$ In die Realencyclopädie hingegen wurde sein Name nicht eingetragen.

2 Vgl. Barron (1964: 211): “There is no indication of the date of Demoteles' tyranny, except that it preceded the war with Megara."

3 So Barron (1964: 211): “This war...must certainly be earlier than the Persian conquest of Ionia."

${ }^{4}$ So Meyer 1931: 185, Loukopoulou 1989: 54, Loukopoulou 2004: 920: "During its early years (c. 570-560), Samian Perinthos faced deadly attacks...from its rival Megarian neighbors."

${ }^{5}$ White (1954: 38): “ca. 600 B.C.”; Figueira (1985: 262): “c. 600”; Shipley (1987: 49, 52 A. 21): "Perhaps... the defence of Perinthus [by the Samians] tied up the Megarian fleet sufficiently to enable Solon to capture Salamis by 594/3." Gehrke (1986: 119) datierte die mit dem Krieg zusammenhängende Revolte gegen die Geomoren "kurz nach 600," es ist indes nicht hundertprozentig sicher, daß die Ereignisse erst in die Zeit nach 600 fallen.
} 
hätte, die neue samische Ansiedlung auszuschalten. ${ }^{6}$ Die Gründung von Perinthos wird in der chronographischen Tradition zumindest kurz vor dem Jahr 600 gesetzt. ${ }^{7}$ Der Krieg wurde also in diesem Raum von gut 50 Jahren (602-546 v. Chr.) geführt.

Der Zeitraum, der für die Alleinherrscahft des Demoteles infrage kommt, umfaßt jedoch noch mehr Jahre. Es steht nämlich nicht fest, daß der Tod des Demoteles in die Zeit nach der Gründung von Perinthos fällt. Auch wissen wir nicht, wie groß der Abstand vom Ende der Regierungszeit des Demoteles zum Ausbruch des Krieges ist. Man darf nicht annehmen, daß Demoteles in diesem Zusammenhang überhaupt nicht erwähnt werden würde, es sei denn, daß er noch kurz vor dem Krieg an der Macht gewesen wäre. Man muß nämlich mit der Möglichkeit rechnen, daß in der samischen Geschichte "die nachdemotelische Zeit" ein Begriff war, der etwa die ganze Zeit vom Tode des Demoteles zum Aufstieg des Syloson bezeichnete. ${ }^{8}$ Es ist dann durchaus möglich, daß Demoteles in "the last third of the seventh century " $z$ u datieren ist. ${ }^{9}$ Kurzum, für die Alleinherrschaft des Demoteles muß man die ganze Zeit von etwa ca. 625 bis ca. 550 in Betracht ziehen.

\section{Die Art der Alleinherrschaft des Demoteles}

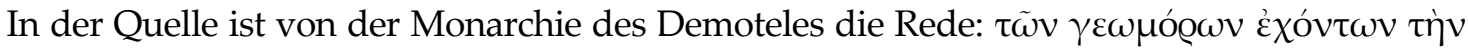

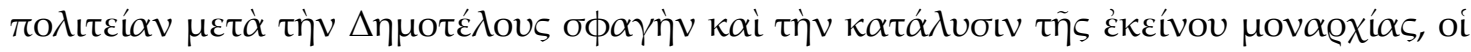

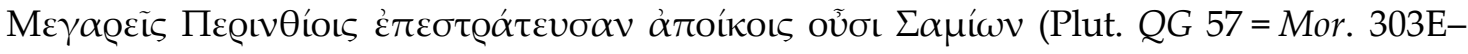
F). ${ }^{10}$ Man hat angenommen, daß hier mit dem Wort $\mu$ ov $\alpha \varrho \chi i ́ \alpha$ die $\beta \alpha \sigma \iota l$ cí $\alpha$ gemeint

\footnotetext{
${ }^{6}$ So Graham (1964: 74): "Megara was no doubt trying to exclude the Samians at the outset from an area where the only competition she was prepared to admit was Milesian."

${ }^{7}$ Zwei geographische Arbeiten führen Perinthos als eine Gründung der Samier auf, ohne das Datum anzu-

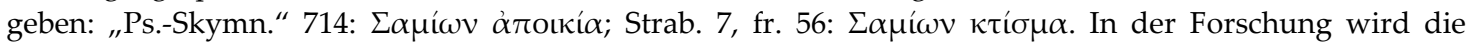
Gründung von Perinthos „,in 602“ (Graham 1982: 120, Loukopoulou 2004: 920), „in c. 602“ (Shipley 1987: 51), "c. 601“ (Bickerman 1980: 168), „um 600“ (Busolt 1893: 470, Beloch 1912: 256, Oberhummer 1937: 805), „c. 600" (Graham 1964: 74) datiert. Verwiesen wird dabei mitunter auf keine Quelle oder auf Hieronymus allein. Das Datum, das als hieronymisch gelten darf, ändert sich jedoch mit der Ausgabe, über die er konsultiert wird. I. I. Scaligerus (Lugduni Batavorum 1606) S. 124 setzte die Gründung auf a. Abr. 1416 (= 601 v. Chr.); A. Schoene (Berlin 1866) S. 91 auf a. Abr. 1413 (=604 v. Chr.), allerdings nach einer Handschr. (F), die Helm „mit Bedacht“ (S. xv) fortgelassen habe; R. Helm (Berlin 1956) S. 98b auf 602 v. Chr. Nach einer Handschr. $(M)$ wurde Perinthos gleichzeitig mit Kamarina gegründet, letztere Stadtgründung wird aber hier auf 601 v. Chr. gesetzt (S. 99b Helm); auch Synkellos erwähnte die Gründung von Kamarina gleich vor

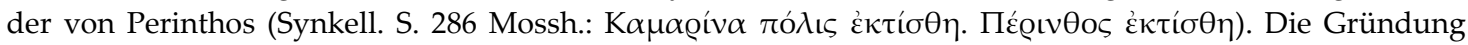
von Kamarina erfolgt zwar anderweitig in den J. 600-596 (Schol. in Pind. Olymp. 5.16, vgl. Thuk. 6.5.3, „Ps.Skymn.“ 294-296), an diesen Stellen wird Perinthos jedoch mit keinem Wort erwähnt.

${ }^{8}$ Nach Berve (1967: 107) mag Sylosons Tyrannis “in die Zeit um 560 fallen"; nach Shipley (1987: 71) gelangte er zur Macht „c. 590.“

${ }^{9}$ Barron 1964: 211.

${ }^{10}$ Zur älteren Überlieferung schreibt Halliday (1928: 212): “The source...may be either Aristotle's Constitution of the Samians or Duris. These...are not, of course, the only possibilities and there is no clue to guide speculation." Okin (1985: 11) behauptet, daß Plutarch zweimal das aristotelische Werk "by title" angebe (Plut. Per. 26, 28); in Wirklichkeit nennt Plutarch an den fraglichen Stellen lediglich Aristoteles, nicht das Werk, das freilich aller Wahrscheinlichkeit nach die Sam. pol. ist. Über das Interesse am Heer des Gottes Dionysos kann Okin (1985: 11-12) indes wahrscheinlich machen, daß QG 56 das Gut des Duris weitergibt; außer von der
} 


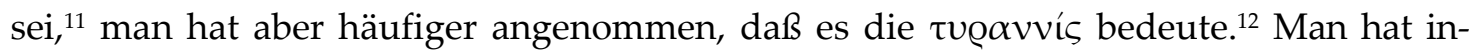
zwischen sogar behauptet, daß es sich hierbei um einen gescheiterten Putschversuch handle. ${ }^{13}$ Letztere Ansicht ist jedoch schlichtweg falsch, denn die einzige Quelle hierzu be-

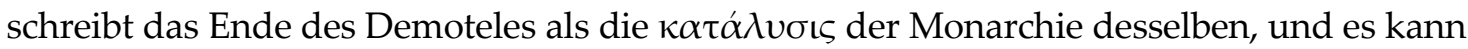
ja eine Auflösung nur dessen stattfinden, was es doch einmal gab. Begründet wurden diese Ansichten zumeist nicht. ${ }^{14}$ Schon der Anbeginn des Zeitraumes, in welchem Demoteles tätig gewesen zu sein scheint, ist aber für einen echten König sehr spät. ${ }^{15}$ Shipley bezog sich zwar nicht explizit auf den griechischen Text, schloß aber trotzdem aus

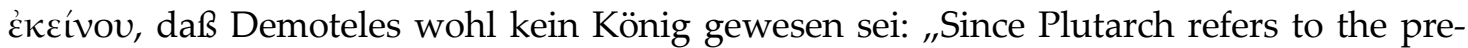
Geomoric regime as his regime, and since it ended when he fell, it was probably a tyr-

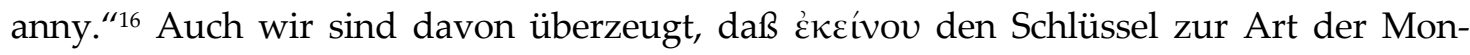
archie darstellt, wir glauben aber, daß Shipley diesem Wort erst ansatzweise gerecht worden ist. ${ }^{17}$

Erstens ist das Wort દ̇ké́vov tatsächlich nicht eindeutig. Shipley hat es offenbar so verstanden, daß es die Herrschaft auf die Person von Demoteles beschränkt und da-

\footnotetext{
Möglichkeit oder Wahrscheinlichkeit, daß Plutarch für QG 54-57, die ja alle Samisches bieten, aus einer Quelle schöpfte, machte Okin (1985: 12-13) über den dramatischen Stil von QG 57 die Urheberschaft des Duris wahrscheinlich. Jacoby (1955: 466) hatte in der Quelle für QG 54-57 „wegen des inhalts und der varianten“ Menodotos von Samos gesehen, der jedoch, wie Okin (1985: 11) bemerkt, bei Plutarch nirgends namentlich genannt ist. Identifiziert wird Menodotos der Samier (Athen. 15.672a) häufig mit Menodotos dem Perinthier (Diod. 26.4), der um 200 v. Chr. tätig war (vgl. Kroll 1931: 900-901), aber der Name ist, wie Jacoby bemerkt, „nicht selten“; Fraser und Matthews (1987: 311) machten aber zugegebenermaßen keinen

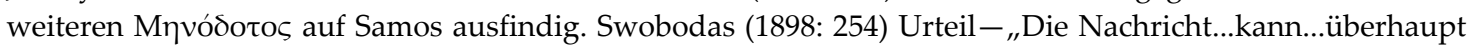

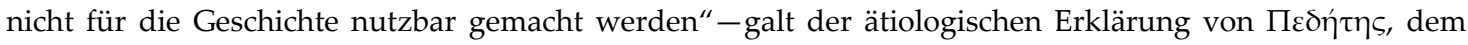
Namen des samischen Andron, betrifft uns also nicht.

${ }^{11}$ Busolt 1893: 316 A. 3, ohne Demoteles namentlich zu nennen, gab die Plutarchstelle als eine das "Ende des Königtums" referierende Quelle an; nach Plaß 1852: 232 war Demoteles „der letzte König“; Ure 1922: 69 A. 5 schreibt: „Demoteles was...the monarch whose murder led to the ascendancy of the geomoroi: he is naturally assumed to have been the last sovereign of the legitimate royal house."

12 Meyer 1893: 614; Beloch 1912: 359; Bürchner 1920: 2214; Barron 1964: 211; Berve 1967: 107; Shipley (1987: 49): "probably a tyranny"; de Libero 1996: 252 A. 6, 411; Rubinstein (2004: 1096): "Demoteles may have been a tyrant whose rule represented an interruption of the oligarchy of the geomoroi."

${ }^{13}$ Mossé (1969: 16): "Comme Cylon, Démotélès échoua et fut mis à mort"; Braccesi (1978: 353): "un tale Demotèles, che-come Cilone in Atene-pagherà con la morte il suo gesto rivoluzionario."

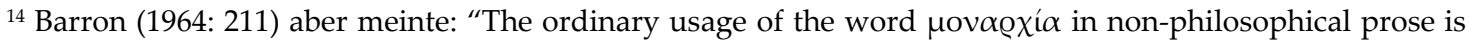

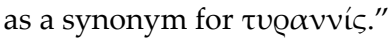

${ }^{15}$ Vgl. Shipley (1987: 39): “Kingships in Greek poleis had mostly come to an end by about 700... There is no evidence as to when exactly the last king of Samos ruled, but it is reasonable to assume that it was around 700 or not many decades later."

${ }^{16}$ Shipley 1987: 49.

${ }^{17}$ Einer der Gutachter betont "the possibility that Plutarch or his source simply did not have any information on the nature of Demoteles' rule" und folgert daraus: "In such a case the opinion of Plutarch or his source must have been a guess or assumption, and the exact meaning of the word ekeinou will not have much importance." Es ist zunächst wenigstens nicht nur denkbar, daß Plutarch es nicht wußte, sondern auch, daß er es doch wußte, es aber an der fraglichen Stelle für ihn ohne Belang war. In beiden Fällen würde

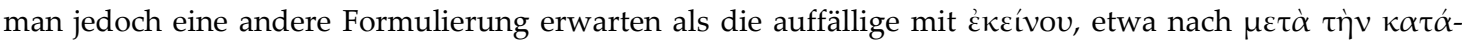

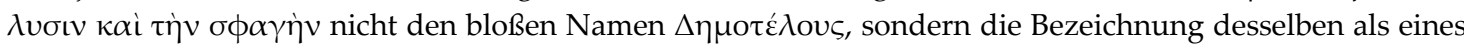

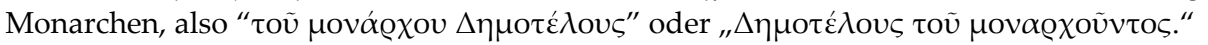




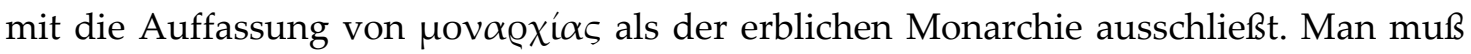
indes berücksichtigen, daß die Quelle nicht zeitgenössisch ist, sie also leicht einen impliziten Vergleich zwischen Demoteles und späteren Herrschern ziehen könnte. In diesem Fall besagt ékcívov, daß mit dem Tod des Demoteles lediglich seine Alleinherrschaft endete, die seine aber nicht das letzte Kapitel in der Geschichte der Alleinherrschaft auf Samos bildete. Diese Auslegung von èké́vov entspricht den Tatsachen, denn-um von anderen samischen Alleinherrschern ganz zu schweigen-der bekannteste der samischen Tyrannen, Polykrates, kam erst in der zweiten Hälfte des sechsten vorchristlichen

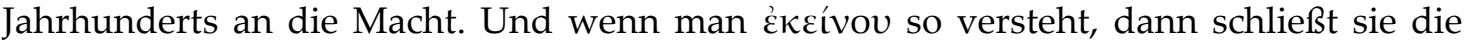
Königsherrschaft nicht mehr aus, denn Plutarch bzw. sein Gewährsmann hätte ohne weiteres einen solchen Vergleich auch zwischen einem König und den späteren Tyrannen anstellen können.

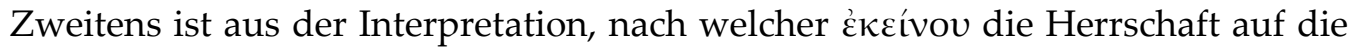
Person von Demoteles beschränkt, eine andere Folgerung abzuleiten als bisher. Es stimmt, daß diese Auslegung von ėké́vou mit der Königsherrschaft unvereinbar ist, da sie erblich war. Die Tyrannis, jedenfalls in ihrer samischen Erscheinungsform, war jedoch ebenfalls erblich. Syloson, der zumeist als ein Vorgänger des Polykrates in der Tyrannis gilt, war mit diesem verwandt; ${ }^{18}$ ob nun Aiakes, der Vater des Polykrates, Tyrann war oder nicht, folgte immerhin dem jüngeren Syloson, dem Bruder des Polykrates (Hdt. 3.39.2), dessen Sohn Aiakes (Hdt. 6.13.1) nach. Da die Forschung nur diese

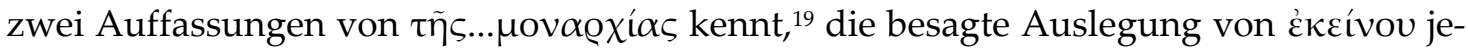

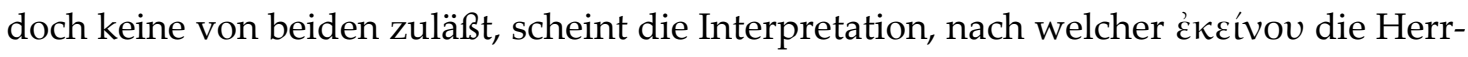
schaft auf Demoteles persönlich beschränkt, gänzlich auszuscheiden. ${ }^{20}$ Zur Erklärung der bewußten Monarchie gibt es aber eine dritte Möglichkeit und zwar eine, die zur Inter-

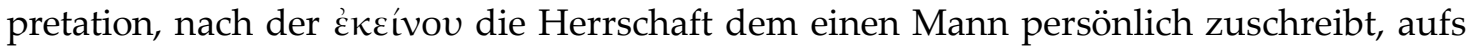

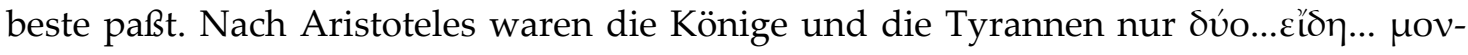

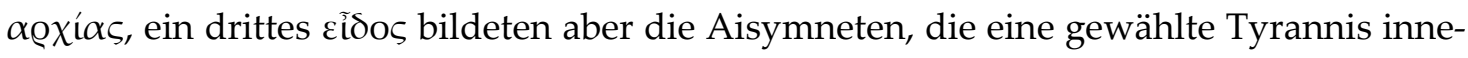

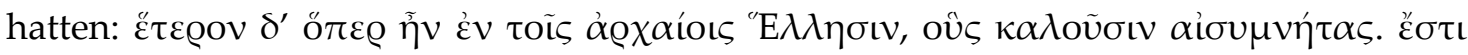

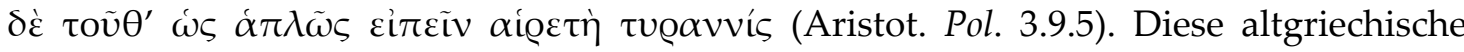
Tyrannis unterscheide sich von der nichtgriechischen in einem Punkt: $\delta \iota \alpha \phi \dot{\varepsilon} \varrho 0 v \sigma \alpha \delta \dot{\varepsilon}$

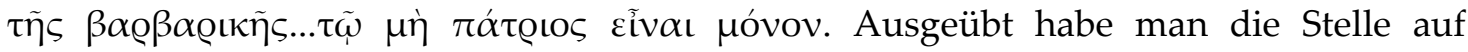
Lebenszeit ( $\delta \dot{\alpha} \beta$ ßíov) oder während einer Frist bzw. bis zur Erledigung einer Aufgabe

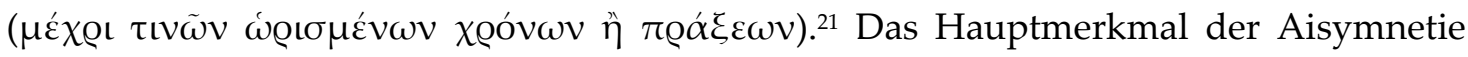

\footnotetext{
${ }^{18}$ Vgl. Shipley (1987: 70): "Evidence for a family connection between them is given by the name of Polycrates' brother... The name Syloson is otherwise unknown."

${ }^{19}$ Vgl. Jacoby (1955: 467): “letzter könig oder tyrann?”

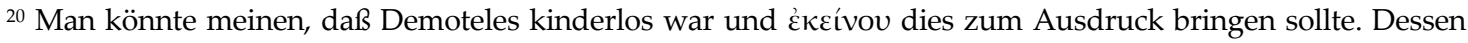
Alleinherrschaft wäre dann im Prinzip zwar erblich, unter den gegebenen Umständen aber es nicht gewesen. Diese Erklärung würde weder die Königsherrschaft noch die Tyrannis ausschließen, sie überzeugt aber nicht, denn es ist wenig wahrscheinlich, daß Plutarch diesen familiären Gegebenheiten Rechnung tragen wollte.

${ }^{21}$ Vgl. Toepffer (1893: 1088): “eine...mit monarchischer Gewalt bekleidete Würde, welche mit Königtum und Tyrannis nahe verwandt ist, nur mit dem Unterschiede, dass sie auf freier Wahl des Volkes beruht...und zur Wiederherstellung oder Sicherung der gestörten oder gefährdeten gesetzlichen Ordnung sei es auf Lebenszeit oder nur auf bestimmte Zeit und bis zur Vollziehung eines bestimmten Auftrags erteilt ward." Nordin
} 
war dann deren Nichterblichkeit, die Eigenschaft also, welche die eine Auslegung von

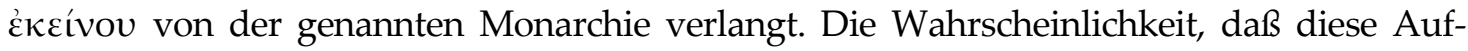

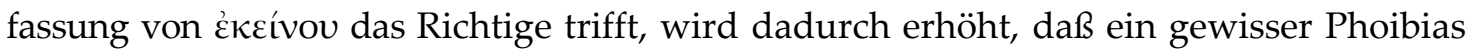
von Samos mit drei anderen-Pittakos von Mytilene, Periandros von Korinth, und einem gewissen Chairemon von Apollonia in Illyricum - als Aisymnetes verzeichnet ist: $\alpha v \theta$ -

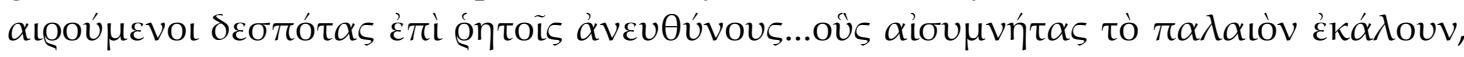

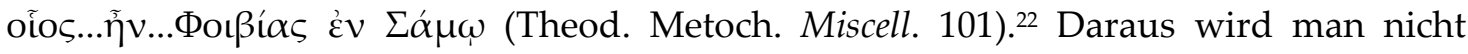

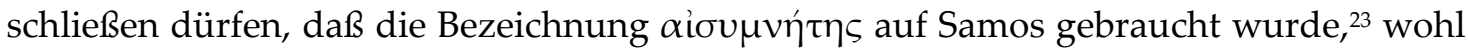
aber, daß den Samiern ein wie auch immer genanntes, aisymnetieähnliches Amt zumindest bekannt war.

Es ist nicht unwahrscheinlich, daß einige, uns nicht zugängliche Quellen Demoteles als Tyrann bezeichneten, andere aber nicht, und daß Plutarch bzw. sein Gewährsmann diesem Problem aus dem Weg ging, indem er von der Monarchie sprach. Und wenn es eine demotelesfeindliche Quelle gab, dann wurde er zweifellos in ihr als Tyrann abgestempelt. Als gesichert darf wohl nur gelten, daß Demoteles kein König gewesen ist. Wenn er nämlich König gewesen wäre, dann hätte er der letzte König von Samos sein müssen, und man würde dann eine anders lautende Todesnachricht erwarten, die betonte, daß damit Samos ein Königtum zu sein aufhörte. Die Art der Monarchie hängt

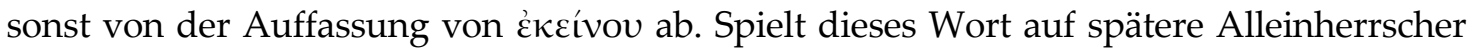
an, dann könnte Demoteles sehr wohl ein Tyrann gewesen sein und war es auch, wenn er denn kein Aisymnetes war. Bedeutet dieses Wort aber, daß die Monarchie des Demoteles nicht erblich war, dann muß er ein Aisymnetes bzw. der Inhaber einer aisymnetieähnlichen Stelle gewesen sein wie sein Landsmann Phoibias. ${ }^{24}$

\author{
Dr. Francis X. Ryan \\ Department of Classics, University of Cincinnati \\ E-mail:fxryan@gmx.de
}

\begin{abstract}
(1905: 406) wollte keinen Unterschied zwischen Tyrannis und Aisymnetie machen. Er fragte: „Woher wissen wir übrigens, daß nicht auch die Aisymnetie ebenso gut wie die Tyrannis bisweilen tatsächlich erblich wurde?" Keine antike Quelle gibt jedoch eine andere Definition als Aristoteles; ein Schüler desselben pflichtet

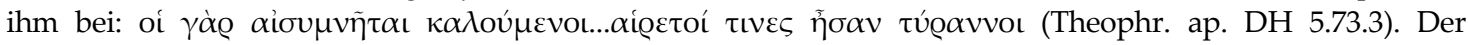
Moment, in dem der Aisymnetes seine Machtstellung vererbt, ist also der Moment, in dem sie eine Aisymnetie zu sein aufhört. Allenfalls könnte man meinen - sieht man einmal von der eigentlichen Machtergreifung abdaß es da, wo die Tyrannis ebenfalls nicht erblich war (bzw. nicht vererbt werden konnte), keinen weiteren Unterschied zwischen ihr und der Aisymnetie gab. Dies trifft aber bekanntlich auf Samos nicht zu.

${ }^{22}$ Der Personenname Фоıßí $\alpha \varsigma$ ist in LGPN 1 weder für Samos noch sonst aufgezeichnet, in der Genitivform Фoıßíov hingegen ist er für Syrakus inschriftlich belegt (IG XIV 52; cf. LGPN 3A.466).

${ }^{23}$ Das Wort ist möglicherweise karischen Ursprungs; so Hegyi 1977: 9-10. Der Titel dürfte in Mytilene gebräuchlich gewesen sein; s. Romer (1982: 41): „Aristotle probably did not invent the use of the term aisymnētēs to describe Pittacus, though he seems to have originated the technical definition of that term. Both because he himself spent some time at Mytilene and because his student Theophrastus came from Eresos on Lesbos, Aristotle had good access to conflicting traditions of local history."

${ }^{24}$ Ein Margo Tytus Forschungsstipendium ermöglichte den zügigen Abschluß dieser Untersuchung.
\end{abstract}




\section{Bibliographie}

Barron, J. P. (1964) ,The Sixth-Century Tyranny at Samos.' - CQ 58, 210-29.

Beloch, K. J. (1912) Griechische Geschichte. 1. Bd., 1. Abt., 2. Aufl. Strassburg: Trübner.

Berve, H. (1967) Die Tyrannis bei den Griechen. München: Beck.

Bickerman, E. J. (1980) Chronology of the Ancient World. Rev. ed. London: Thames and Hudson.

Braccesi, L. (1978) 'Le tirannidi e gli sviluppi politici ed economico-sociali.' - Bandinelli, R. B. (ed.), Storia e civiltà dei Greci. Milano: Valentino Bompiani, 2.329-82.

Bürchner, L. (1920) 'Samos 4.' - RE 1A, 2162-2218.

Busolt, G. (1893) Griechische Geschichte bis zur Schlacht bei Chaeroneia. 1 Bd., 2. Aufl. Gotha: Perthes.

Figueira, T. J. (1985) ,Chronological Table: Archaic Megara, 800-500 B.C.' - Figueira, T. J.; Nagy, G. (eds.), Theognis of Megara: Poetry and the Polis. Baltimore: Johns Hopkins University Press, 261-303.

Fraser, P. M.; Matthews, E. (eds.) $(1987,1997)$ A Lexicon of Greek Personal Names. Vol. 1: 1987. Vol. 3A: 1997. Oxford: Clarendon Press.

Gehrke, H. (1986) Jenseits von Athen und Sparta. Das Dritte Griechenland und seine Staatenwelt. München: Beck.

Graham, A. J. (1964) Colony and Mother City in Ancient Greece. New York: Barnes and Noble.

Graham, A. J. (1982) 'The Colonial Expansion of Greece.' - Boardman, J.; Hammond, N. G. L. (eds.), The Cambridge Ancient History, vol. 3, 2nd ed. Cambridge: University Press, 83-162.

Halliday, W. R. (1928) The Greek Questions of Plutarch. Oxford: Clarendon Press.

Hegyi, D. (1977) ‘Der Ursprung der Aisymneteia.' - ACD 13, 7-10.

Jacoby, F. (1955) Die Fragmente der griechischen Historiker, 3B: Kommentar (Text) und 3B: Kommentar (Noten). Leiden: Brill.

Kroll, W. (1931) ,Menodotos 1.' - RE 15, 900-901.

LGPN - s. Fraser \& Matthews 1987, 1997.

Libero, L. de (1996) Die archaische Tyrannis. Stuttgart: Steiner.

Loukopoulou, L. D. (1989) Contribution à l'histoire de la Thrace propontique durant la période archaïque. Athena: K.E.R.A.

Loukopoulou, L. (2004) 'Perinthos.' - Hansen, M. H.; Nielsen, T. H. (eds.), An Inventory of Archaic and Classical Poleis. Oxford: University Press, 919-21.

Meyer, Eduard (1893) Geschichte des Altertums. 2. Bd. Stuttgart: Cotta

Meyer, Ernst (1931) ,Megara 2.' - RE 15, 152-205.

Mossé, C. (1969) La Tyrannie dans la Grèce antique. Paris: Presses Universitaires de France.

Nordin, R. (1905) 'Aisymnetie und Tyrannis.' - Klio 5, 392-409.

Oberhummer, E. (1937) 'Perinthos 1.' - RE 19, 802-13.

Okin, L. A. (1985) 'Theognis and the Sources for the History of Archaic Megara.' Figueira, T. J.; Nagy, G. (eds.), Theognis of Megara: Poetry and the Polis. Baltimore: Johns Hopkins University Press, 9-21. 
Plaß, H. G. (1852) Die Tyrannis in ihren beiden Perioden bei den alten Griechen. 1. Theil. Bremen: Schlodtmann.

Romer, F. E. (1982) ,The Aisymnēteia: A Problem in Aristotle's Historic Method.' - AJP 103, 25-46.

Rubinstein, L. (2004) 'Samos.' - Hansen, M. H.; Nielsen, T. H. (eds.), An Inventory of Archaic and Classical Poleis. Oxford: University Press, 1094-98.

Shipley, G. (1987) A History of Samos, 800-188 BC. Oxford: Clarendon Press.

Swoboda, H. (1898) 'Zur Verfassungsgeschichte von Samos.' - Festschrift für Otto Benndorf zu seinem 60. Geburtstage. Wien: Hölder, 250-55.

Toepffer, J. (1893) ,Aisymnetes 1.' - RE 1, 1088-91.

Ure, P. N. (1922) The Origin of Tyranny. Cambridge: University Press.

White, M. (1954) 'The Duration of the Samian Tyranny.' - JHS 74, 36-43. 